\title{
A Study on Machine Learning Approach for Fingerprint Recognition System
}

\author{
Aayushi Tamrakar \\ M.Tech. Scholar \\ Department of CSE \\ Technocrats Institute of Technology \\ Bhopal, India \\ tamrakarayushi@gmail.com
}

\author{
Neetesh Gupta \\ Professor \\ Department of CSE \\ Technocrats Institute of Technology \\ Bhopal, India \\ gupta_neetesh81@yahoo.com
}

\begin{abstract}
A biometric system is an evolving technology that is used in various fields like forensics, secured area and security system. Authentication system like fingerprint recognition is most commonly used biometric authentication system. Fingerprint method of identification is the oldest and widely used method of authentication used in biometrics. There are several reasons like displacement of finger during scanning, environmental conditions, behavior of user, etc., which causes the reduction in acceptance rate during fingerprint recognition. The result and accuracy of fingerprint recognition depends on the presence of valid minutiae. Fingerprint Recognition system designed uses various techniques in order to reduce the False Acceptance Rate (FAR) and False Rejection Rate (FRR) and to enhance the performance of the system. This paper reviews the fingerprint classification including feature extraction methods and learning models for proper classification to label different fingerprints. A comparative study of different recognition technique along with their limitations is also summarized and optimum approach is proposed which may enhance the performance of the system.
\end{abstract}

Keywords-Biometric system; Fingerprint Recognition System; Minutiae; Reconstruction; Feature Extraction; Classification;

\section{INTRODUCTION}

Biometric systems are now commonly used in different parts of everyday life such as building access and computer login. Fingerprint detection is one of the most prevalent methods for personal identification within all biometric systems [1]. Throughout the world, fingerprint recognition is accepted by a large part of the population because of its fast, secure, and easy way of personal identification.

Fingerprint is the most interesting and oldest human identity used for recognition of individual. In the early twentieth century, fingerprint was formally accepted as valid signs of identity by law-enforcement agencies.

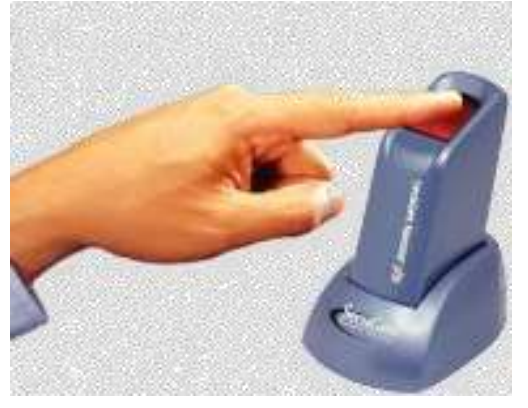

Figure 1: Fingerprint Scanner

On the basis of this the automatic fingerprint recognition system for authentication and identification i.e. Basically there are two types of fingerprint Recognition System AFAS (Automatic Fingerprint Authentication System), AFIS (Automatic Fingerprint Identification/Verification System) developed by the scientist and developers recently $[2,3]$.

Unique finger impression acknowledgment framework depends on two essential feature such as:

- Persistence: The fundamental attributes of unique finger impression don't change with time.

- Individuality: The finger impression is unique for each person.

The main parameters characterizing a digital fingerprint image are such as Resolution, Area, No. of pixels, Depth, Geometric accuracy, etc.

Typically, fingerprints consist of ridge and valley patterns on the tips of human fingers. Ridges (also called ridge lines) are dark whereas valleys are bright (see Figure 2). Ridges and valleys often run in parallel; sometimes they bifurcate and sometimes they terminate [5]. Thanks to their uniqueness and continuity, the use of fingerprints is considered to be one of the most reliable methods of personal verification. 


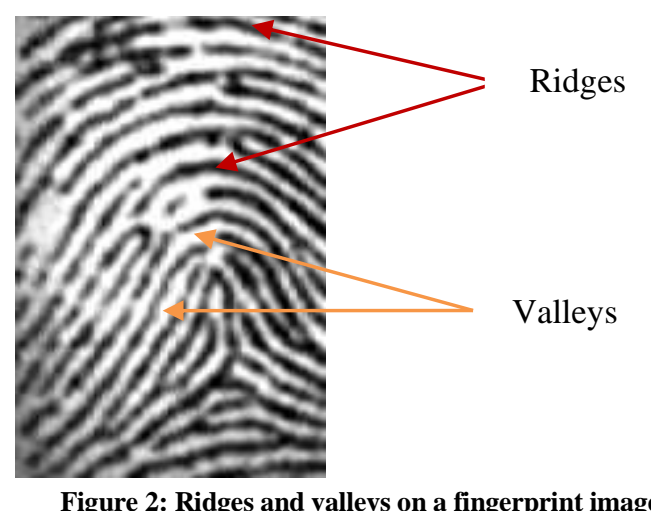

Figure 2: Ridges and valleys on a fingerprint image

When analyzed at the global level, the fingerprint pattern exhibits one or more regions where the ridge lines assume distinctive shapes (characterized by high curvature, frequent termination, etc.). These regions (called singularities or singular regions) may be classified into three typologies: loop, delta, and whorl (see Figure 3).

Fingerprint identification is popular because of its various characteristics such as: ease in collecting the data, easily accessible numerous sources (ten fingers available for collection). The use of fingerprints has a past record from the nineteenth century. It is during this time Sir Francis Galton defined the characteristics points of the fingerprints with which it can be identified and these were referred to as Galton points [6]. These Galton Points are the base on which fingerprint recognition system is built. The subset of Galton points are used to represent the fingerprint image and these are referred to as minutiae. Minutia refers to various ways that the ridges can be discontinuous. For example, a ridge can suddenly come to an end (termination), or can divide into two ridges (bifurcation).

Most of the fingerprint recognition and classification algorithms require a feature extraction stage for identifying salient features. The features extracted from fingerprint images often have a direct physical counterpart (e.g., singularities or minutiae), but sometimes they are not directly related to any physical traits (e.g., local orientation image or filter responses). Features may be used either for matching or their computation may serve as an intermediate step for the derivation of other features. For example, some preprocessing and enhancement steps are often performed to simplify the task of minutiae extraction [7].

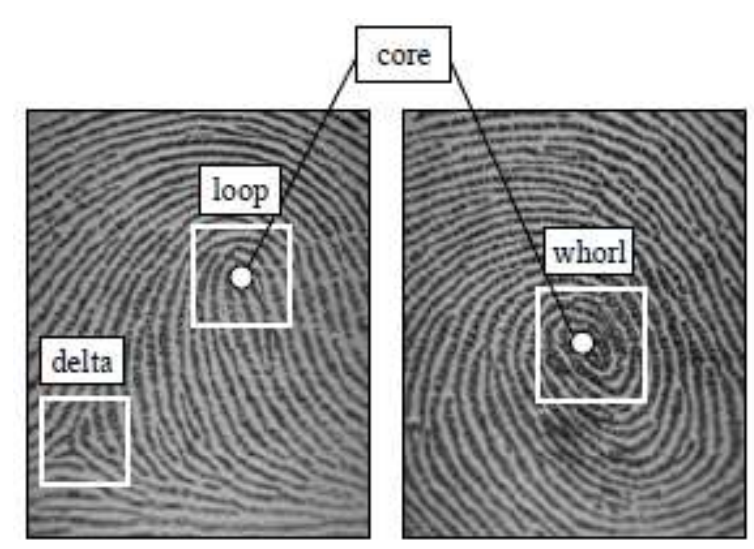

Figure 3: Singular regions and core points in fingerprint images

Organization of the rest of the paper is as follows: Section II discusses about the detailed review of the related work done in the field of fingerprint recognition. Section III discusses about the optimum approach for a fingerprint recognition system. Section IV discusses the conclusion and future work to be carried on to improve the performance of the system.

\section{RELATED WORK}

Pavithra [1] presented a complete crime scene fingerprint identification system using deep machine learning with Convolutional Neural Network (CNN). Images are acquired from crime scene using methods ranging from precision photography to complex physical and chemical processing techniques and saved as the database. The images collected from the crime scene are usually incomplete and hence difficult to categorize. Suitable enhancement methods are required for pre-processing the fingerprint images. Minutiae are extracted from the fingerprint images. The features of preprocessed data are fed into the $\mathrm{CNN}$ as input to train and test the network. The experimental results demonstrated on database using Open CV-Python shows high accuracy of $80 \%$ recognition an partial or full fingerprints in the criminal database.

B. Wenxuan [2] proposed an algorithm which can effectively improve the location accuracy of the edge points in the location area. By incorporating neighborhood clustering into positioning reference, the method improves the positioning accuracy of edge positioning points, improves the overall indoor positioning effect, and improves the positioning accuracy to a certain extent. The simulation environment is built to verify the proposed algorithm. The experimental results show that the improved algorithm improves the positioning accuracy of edge locating points to a certain extent, and improves the overall positioning accuracy of edge locating points. 
O. I. Abiodun [3] furnishes readers with a clearer understanding of the current, and new trend in ANN models that effectively addresses PR challenges to enable research focus and topics. Similarly, the comprehensive review reveals the diverse areas of the success of ANN models and their application to PR. In evaluating the performance of ANN models, some statistical indicators for measuring the performance of the ANN model in many studies were adopted. Such as the use of mean absolute percentage error (MAPE), mean absolute error (MAE), root mean squared error (RMSE), and variance of absolute percentage error (VAPE). The result shows that the current ANN models such as GAN, SAE, DBN, RBM, RNN, RBFN, PNN, CNN, SLP, MLP, MLNN, Reservoir computing, and Transformer models are performing excellently in their application to PR tasks. Therefore, the study recommends the research focus on current models and the development of new models concurrently for more successes in the field.

Serafim [4] presented a technique of segmentation of the region of interest based on convolutional neural networks (CNN) without pre-processing steps. The new approach was evaluated in two different architectures from state of the art, presenting similarity indexes Distance of Hausdorff (5.92), Dice coefficient (97.28\%) and Jaccard Similarity (96.77\%) superior to the classic methods. The error rate $(3.22 \%)$ was better than five segmentation techniques from state of the art and showed better results than another deep learning approach, presenting promising results to identify the region of interest with potential for application in systems based on biometric identification.

Han [5] proposed a method based on adaptive median filter for fingerprint image enhancement processing and impulse noise removal in the paper. The use of adaptive median filtering to remove the impulse noise of the fingerprint image mainly involves three steps. First, the size of the adaptive median filter window is initialized, and it is judged whether the center pixel of the filter window in the fingerprint image is impulse noise. Second, the size of the filter window is determined based on the median value, the maximum value, and the minimum value within the filter window. Finally, median filtering is performed on the fingerprint image under the filter window size obtained in the previous steps, and the filter output value is used instead of the window center pixel value. The method is tested on rolled fingerprint images contaminated by impulse noise and fingerprint images contaminated by impulse noise from a crime scene.
Experimental results show that the method based on adaptive median filter for fingerprint image enhancement outperforms the traditional median filtering method in filtering impulse noise performance.

Rezaei [6] explored an efficient algorithm for fingerprint recognition based on hybrid features of Discrete Cosine Transform (DCT), Discrete Wavelet Transform (DWT), and moment methods. The proposed technique can be applied for high accuracy fingerprint recognition task in biometric systems.

\section{III.EXISTING TECHNOLOGIES FOR FINGERPRINT ANALYSIS}

A fingerprint is characterized by abundant and strong textural information. The textural properties of a live fingertip surface are dependent upon skin elasticity, pore distribution and perspiration phenomenon. As a result, the pixels along and around the ridges of a live fingerprint exhibit wide and random variations in gray-level values. The material and physical characteristics of spoof fingers are constant. The live and spoof fingerprints differ in ridge width, inter-ridge distances, ridge frequency and gray-level distribution [16]. Therefore, texture features-based methods which can capture these variations from fingerprint image properties are expected to perform better.

Spatial domain pixel intensity value variations create texture patterns in an image. One of the applications of image texture is image analysis using texture properties. The existing texture features-based fingerprint liveness detection methods are grouped into following categories:
i. Global texture features
ii. Local texture features
iii. Hybrid (global and local) texture features

\section{IV.APPLICATION OF FINGERPRINT IDENTIFICATION}

Fingerprints in the crime scene plays an important role to identify the criminal involved in the crime. Crime scene images (CSI) are images taken from the crime spot. When crime is occurred, the investigator takes both latent and patent sample of fingerprints left behind. The patent fingerprints are visible by naked eye, so they are simply photographed. But latent fingerprints [1] are invisible and these samples are more difficult to perceptible. These samples can be lifted through different techniques. The use of cyanoacrylate vapors which sticks to prints and make them visible in the present of normal light. This 
method is much difficult, so normally in crime scene, the investigators apply a [2] fine dusting powder (aluminium dust or black granular) to the surface in which fingerprints to be extracted. The dust actually sticks to the fingerprint then they use clear tape to lift the fingerprint. After the lifting the fingerprints, the prints are scanned and saved in the digital image form. The fingerprints taken from the crime scene is unintentionally made and these images are noisy or partial prints and difficult to identify.

\section{CONCLUSION}

In this paper, we have shown different methods and techniques which can be used to identify a person through its fingerprint. These mentioned methods conclude that the fingerprint is fast and accurate for more reliable and secure system. Future research work can be carried out to improve the quality of the image by improving the image enhancement technique and develop a better matching technique. Taking advantage of the segmentation features, learning capability of classifier, the future work will be focused to achieve an efficient detection rate for partial fingerprint by applying deep learning approach.

\section{REFERENCES}

[1] Pavithra. R and K.V. Suresh, "Fingerprint Image Identification for Crime Detection", IEEE International Conference on Communication and Signal Processing, April 4-6, 2019, pp. 0797-0800.

[2] B. Wenxuan, F. Zhihong, P. Min and W. Pu, "Research on Indoor Edge Location Based on Location Fingerprint Recognition," International Conference on Measuring Technology and Mechatronics Automation (ICMTMA), Qiqihar, China, 2019, pp. 302-306.

[3] O. I. Abiodun et al., "Comprehensive Review of Artificial Neural Network Applications to Pattern Recognition," in IEEE Access, vol. 7, pp. 158820-158846, 2019.

[4] P. B. S. Serafim, "A Method based on Convolutional Neural Networks for Fingerprint Segmentation," International Joint Conference on Neural Networks (IJCNN), Budapest, Hungary, 2019, pp. 1-8.

[5] K. Han, Z. Wang and Z. Chen, "Fingerprint Image Enhancement Method based on Adaptive Median Filter," 2018 24th Asia-Pacific Conference on Communications (APCC), Ningbo, China, 2018, pp. 40-44.

[6] Z. Rezaei and G. Abaei, "A Robust Fingerprint Recognition System Based on Hybrid DCT and DWT," 2017 24th National and 2nd International Iranian Conference on Biomedical Engineering (ICBME), Tehran, 2017, pp. 330333.

[7] H. Su, K. Chen, W. J. Wong and S. Lai, "A deep learning approach towards pore extraction for high-resolution fingerprint recognition," IEEE International Conference on Acoustics, Speech and Signal Processing (ICASSP), New Orleans, LA, 2017, pp. 2057-2061. 